\title{
Thirteen-Month-Old Boy with Malignant Lymphoma Having Symptoms Mimicking Acute Otitis Media and Mastoiditis with Facial Palsy
}

\author{
Sho Kanzaki ${ }^{a}$ Hideyuki Saito ${ }^{a}$ Tetsuya Mori ${ }^{b}$ Noriko Shimasaki ${ }^{b}$ \\ Makio Mukaic Kaoru Ogawa ${ }^{\text {a }}$ \\ Departments of a Otolaryngology, Head and Neck Surgery, ${ }^{b}$ Pediatrics and ${ }^{\mathrm{C}}$ Pathology, Keio University School of \\ Medicine, Tokyo, Japan
}

\section{Key Words}

Malignant lymphoma · Mastoiditis · Pediatrics · Facial palsy

\begin{abstract}
Non-Hodgkin diffuse large B-cell lymphomas of the mastoid that extend from the nasopharynx are extremely rare in children. Moreover, in lymphoproliferative diseases, the presence of otoneurological signs prior to systemic disease involvement is rare. Here, we present a rare case of non-Hodgkin B-cell lymphoma invading the middle ear and mastoid in a 1-year-old boy that mimicked acute mastoiditis with complete facial nerve palsy. As this case illustrates, physicians should consider a diagnosis of malignant lymphoma if a patient presents with otitis media and mastoiditis accompanied by facial palsy.

Copyright $\odot 2011$ S. Karger AG, Basel
\end{abstract}

\section{Introduction}

Non-Hodgkin lymphomas (NHLs) of the mastoid that extends from the nasopharynx are extremely rare in children. Most of the childhood NHLs are subdivided into Burkitt's lymphoma, diffuse large B-cell lymphoma (DLBCL), lymphoblastic lymphoma and anaplastic large- cell lymphoma. DLBCL accounts for $10-20 \%$ of childhood NHL [1]. DLBCL can spread to all areas of the body, including the head and neck. Involvement of the temporal bone as part of generalized lymphoma has been reported; however, clinical evidence of temporal bone or middle-ear involvement is unusual $[2,3]$. Here, we present a rare childhood case of DLBCL invading the middle ear and mastoid that mimicked acute mastoiditis with complete facial nerve palsy. NHLs are further subdivided into T-cell, B-cell, low-grade and high-grade lymphomas based on immunohistochemical criteria [4]. Both types originate most commonly within a lymph node. Extranodal presentation is most common with diffuse largecell malignant lymphoma [4].

\section{Case Report}

This is the case of a 13-month-old boy who presented to our clinic with complete seventh cranial nerve palsy. The boy was finally diagnosed as having DLBCL with mastoid infiltration. Six months prior to the onset of facial palsy, another clinic had diagnosed the patient with repeated otitis media twice. Thus, at that time, the boy had been treated with several antibiotics, including Sawacillin or Augmentin (amoxicillin and clavulanate) or Meiact (a third-generation cephalosporin antibiotic).

\section{KARGER}

Fax +41613061234 E-Mail karger@karger.ch www.karger.com
(C) 2011 S. Karger AG, Basel

0301-1569/11/0735-0266\$38.00/0

Accessible online at:

www.karger.com/orl
Sho Kanzaki, MD, PhD

Department of Otolaryngology, Head and Neck Surgery

Keio University School of Medicine, 35 Shinanomachi, Shinjuku

Tokyo 160-0082 (Japan)

Tel. +81 35363 3827, E-Mail skan@a7.keio.jp 
Fig. 1. Temporal bone CT before surgery. CT demonstrated soft tissue density (black arrowheads) in the right tympanomastoid cavity (a) and the eroded eustachian tube (b).

Fig. 2. Perioperative findings. a The tympanic membrane (arrowhead) could not be visualized because of persistent bleeding. b Granulation and associated bleeding (arrowhead) were observed in the inner parts of the middle ear.
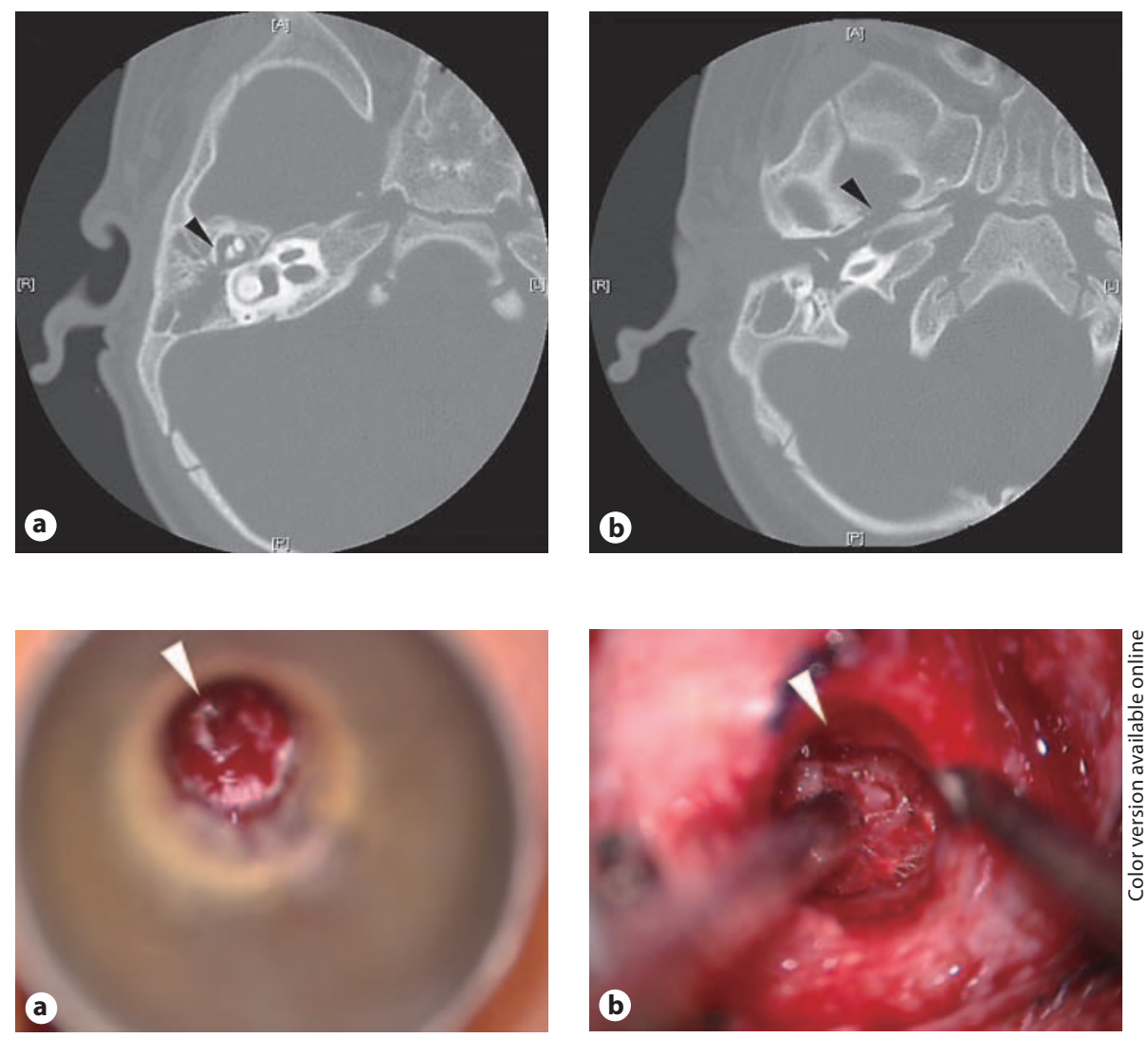

On X (month) 16, Y (year), the patient visited an emergency room, presenting with right facial palsy, otorrhea and a fever of $37.7^{\circ} \mathrm{C}$. Although his right tympanic membrane was swollen, the right postauricular membrane was not. Myringotomy of the swollen tympanic membrane revealed a little purulent discharge associated with methicillin-resistant Staphylococcus aureus infection, which was sensitive to arbekacin. We suspected acute mastoiditis (right side) accompanied by facial palsy. On admission, we initiated intravenous antibiotics, which included arbekacin, without steroids. Five days later, CT of the temporal bones revealed a soft tissue mass in the mastoid cavity and eroded eustachian tube (fig. 1). Because the 5-day treatment of antibiotics was ineffective, we performed mastoidectomy (right side) to remove a possible abscess and to decompress the facial nerve. During surgery, we noted that the tympanic membrane was red and swollen (fig. 2). Neither abscess nor cholesteatoma was found. Instead, the mastoid cavity and middle ear were full of granulation tissue, but no pus. Some of the mastoid granulations were biopsied for histopathological analysis, which showed that the mastoid cavity contained mostly necrotized tissue with infiltration of atypical B lymphocytes, suspicious of malignant lymphoma.

Thereafter, CT demonstrated a swollen cervical lymph node and nasopharynx. $\mathrm{T}_{1}$-weighted magnetic resonance imaging (MRI) also revealed a tumor in the nasopharynx invading that parapharyngeal space and middle cranial base (fig. 3). The tumor may have originated primarily from adenoid lymphoid tissue. On $\mathrm{X} \pm 1$ (month) 5, Y (year), the second biopsy was performed, this time on the cervical lymph node and nasopharynx. The biopsy specimen showed diffuse infiltration of large immature cells expressing CD20 and CD79 $\alpha$ (fig. 4). These cells were negative for TdT, CD10 or CD3. The MIB-1 index of these cells was $60 \%$. The pathologic findings were compatible with DLBCL; however, Epstein-Barr-virus-encoded small-RNA in situ hybridization was positive in lymphoma cells. According to the Murphy classification, the disease was determined to be stage II, because no other involved site including bone marrow or cerebrospinal fluid could be identified.

After the first course of chemotherapy with prednisolone/ dexamethasone, vincristine, cyclophoshamide, high-dose methotrexate, pirarubicin and intrathecal therapy, partial response of the lymphoma was observed. However, after the second course of chemotherapy, the size of the tumor was significantly enlarged by CT imaging. Although the boy received salvage chemotherapy including rituximab, ifosphamide, carboplatin and etoposide, the tumor kept enlarging. After the second course of the salvage chemotherapy, the parents of the boy hoped for palliative treatment. Due to bleeding from the enlarged tumor, the patient died of lymphoma 10 months after the onset of facial palsy. 

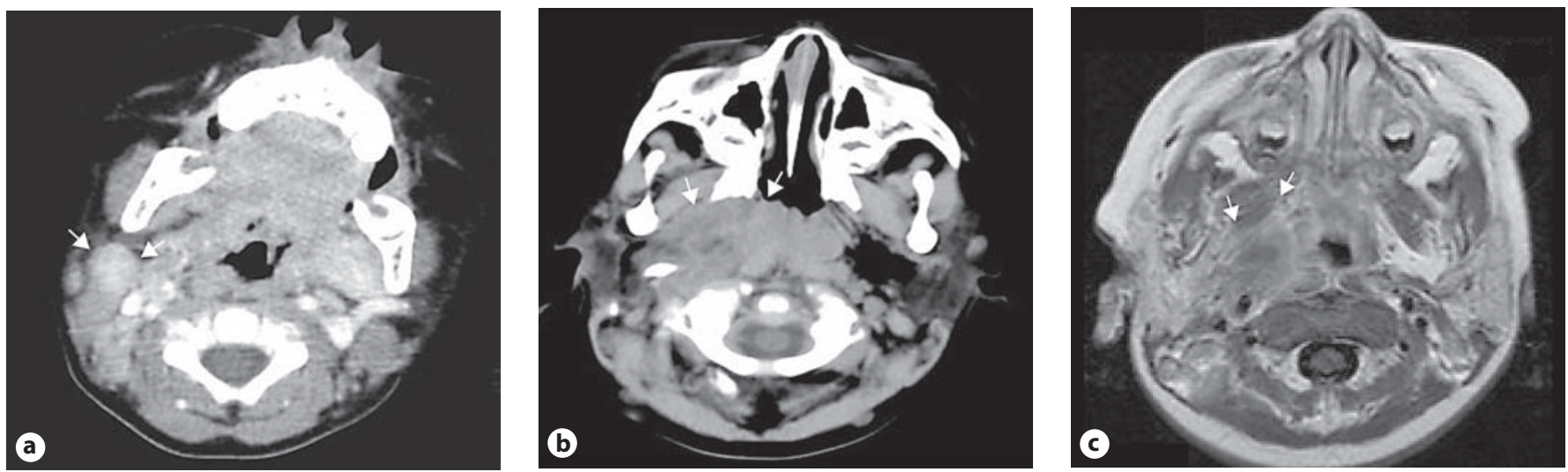

Fig. 3. The neck CT and the neck MRI performed after mastoidectomy. a, b Neck CT; the arrows indicate the swollen lymph node on the right side of the upper neck (a) and epipharynx (b). c Neck MRI; the arrows indicate that the tumor invaded the parapharyngeal space.
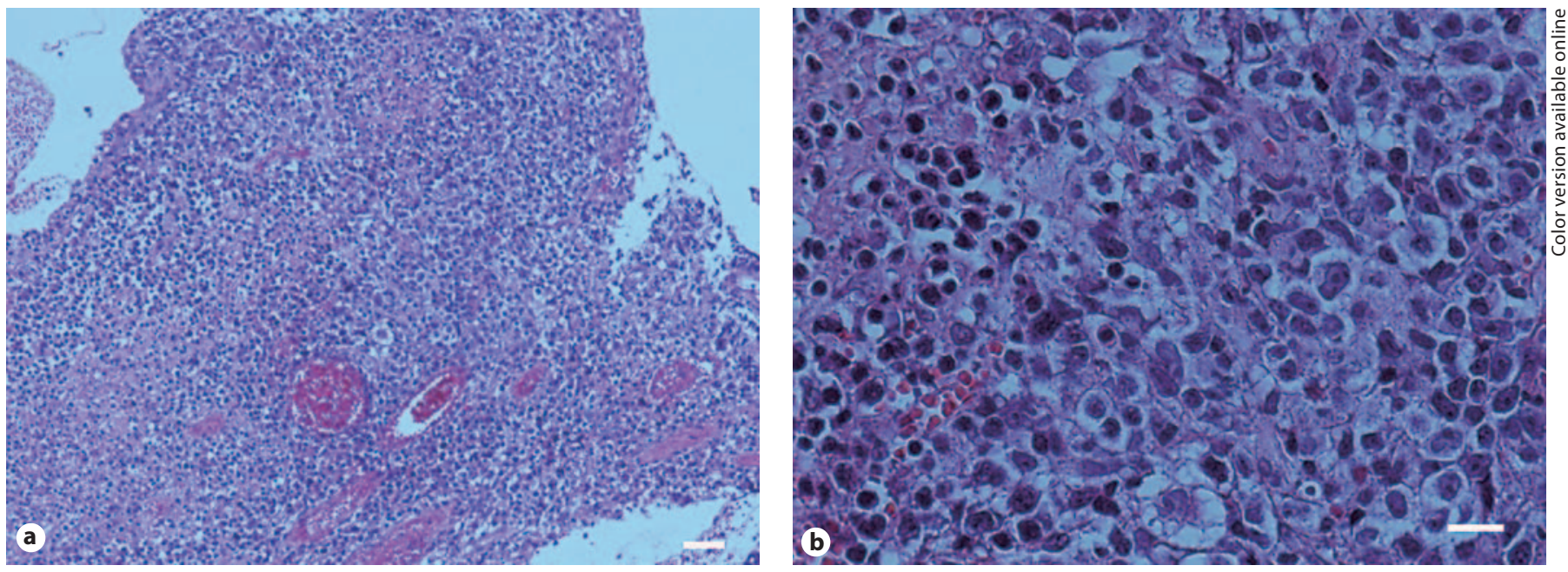

Fig. 4. Immunohistopathology of adenoid tissue. a HE staining (lower magnification). Scale bar $=50 \mu \mathrm{m}$. b HE staining (higher magnification). Scale bar $=10 \mu \mathrm{m}$. c Immunohistological analysis. Scale bar $=10 \mu \mathrm{m}$. Immunohistopathological analysis of biopsied tissues demonstrated malignant DLBCL with the following B-cell marker profile: CD20(+), CD79 $\alpha(+)$, UCHL1(-), CD3(-).

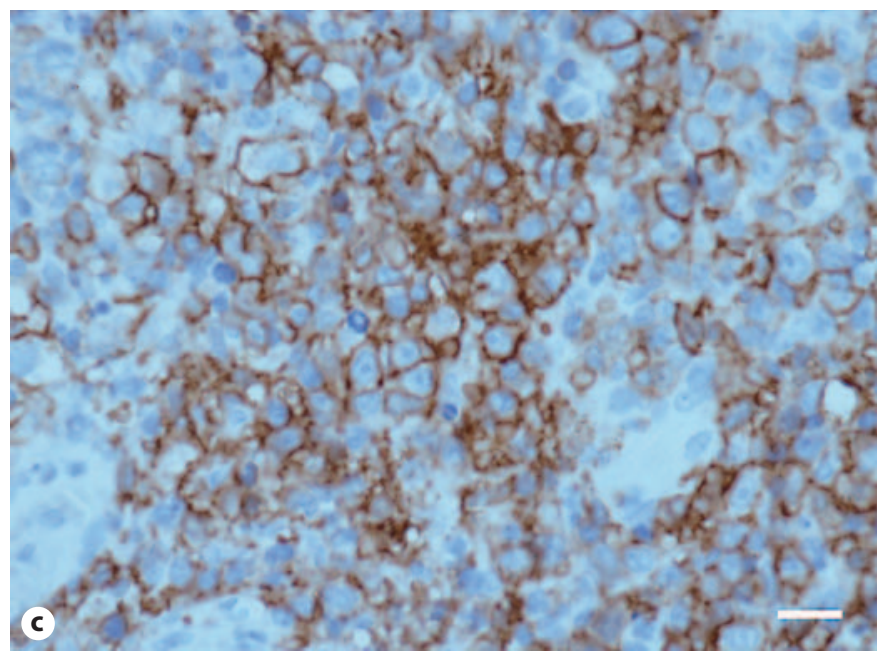




\section{Discussion}

This is a rare case of pediatric B-cell NHL of the middle ear and mastoid. The lymphoma extended from the deep side of the nasopharynx, and the patient presented with facial palsy mimicking acute middle ear infection and mastoiditis. As facial palsy is not a well-recognized symptom of childhood lymphoma, as shown in this case, lymphoma of the mastoid can be misdiagnosed as middle ear infection and mastoiditis. Although the initial symptoms of this type of NHL resemble those of a middle ear infection, antibiotics do not resolve these symptoms [5].

The literature documents 1 case of Hodgkin's disease of the middle ear and mastoid [6]. In addition, we found 2 reports of children presenting with facial nerve palsy associated with middle ear disease, one of an immunocompetent 2-year-old male with spontaneous regression of an Epstein-Barr-virus-associated monoclonal lymphoid proliferation [7] and the second of a 5-year-old male with facial nerve palsy secondary to primary NHL of the middle ear [5]. Thus, as these and the present case illustrate, children presenting with facial nerve palsy and conductive hearing loss should be evaluated by CT in order to exclude temporal bone malignancies [5]. In our 1-year-old patient, bone destruction of the skull base or mastoid margin was not demonstrated in CT images. This often suggests that the mass occupying the mastoid is not malignant. However, our patient did present with symptoms that are most commonly reported in patients with clinical manifestations of middle-ear lymphoma: otalgia, moderate-to-severe conductive hearing loss, lowgrade fever and facial paralysis $[8,9]$.

With the present case, there are 4 issues to consider regarding the diagnosis and treatment of NHL of the mastoid. First, physicians need to consider possible malignancies for patients presenting with otitis media and facial palsy. In this case, we speculated that the lymphoma originated in either the nasopharynx or Waldeyer's ring and extended to the parapharyngeal space and middle ear through the eustachian tube. This is consistent with our patient's repeated intermittent episodes of earache, which was most likely caused by a blocked eustachian tube, infection and necrotizing tissue in the middle ear.

Second, as demonstrated in the present case, NHL symptoms can mimic those of acute otitis media and mastoiditis. Thus, we emphasize the importance of biopsying the mastoid mucosa of patients with acute mastoiditis. Obtaining biopsy tissue was difficult in the present case, because most of the tumors were located within ne- crotic tissue in the mastoid and because of the presence of responsive inflammation, which was confirmed by histopathology of neck lymph nodes. In addition, because the mass was located in deep portions of the nasopharynx or adenoid tissue, we had to approach the biopsy site blindly. Eventually, we were able to access the pathological tissue, but the size of the biopsy sample was small.

Third, arriving at a pathological diagnosis was difficult not only because of an extremely small biopsy specimen, but also because other diseases affecting the mastoid, such as T-cell lymphomas, are commonly associated with facial palsy $[10,11]$, which could possibly mask the real problem. Thus, the use of immunophenotyping was crucial in the diagnosis of this case.

Fourth, since childhood lymphomas of the mastoid are rare and sporadic, the prognosis of this disease is unknown, and its treatment has not been established. Laskar et al. [12] investigated the prognostic factors and treatment outcome of 32 children with NHL of Waldeyer's ring. They found that the age of the patient when diagnosed, the size of the tumor and the presence of $\mathrm{B}$ symptoms (fever of $38^{\circ} \mathrm{C}$ or higher, night sweats or unexplained weight loss of $10 \%$ or more [13]) significantly influenced the outcome of children with NHL of Waldeyer's ring [12]. The same group also found that combined treatment (chemotherapy and radiotherapy) had better outcomes than chemotherapy alone [12]. In the present case, none of the chemotherapy approaches were successful. As more cases of childhood NHL of the mastoid come to the fore, the prognosis and treatment of this disease will become clearer.

In summary, facial palsy could occasionally be a symptom of childhood lymphoma, which can be misdiagnosed as acute mastoiditis. Therefore, physicians should consider a diagnosis of malignant lymphoma if a patient presents with otitis media and mastoiditis accompanied by facial palsy.

\section{Acknowledgements}

We would like to thank Dr. Y. Momoshima of the Department of Radiology, Keio University School of Medicine, for his helpful assistance. This work was supported by a Grant-in-Aid for Scientists (21791643 to S.K.)

ORL 2011·73:266-270 269 


\section{References}

1 Burkhardt B, Zimmermann M, Oschlies I, Niggli F, Mann G, Parwaresch R, et al: The impact of age and gender on biology, clinical features and treatment outcome of nonHodgkin lymphoma in childhood and adolescence. Br J Haematol 2005;131:39-49.

$>2$ Fierstein J, Thawley S: Lymphoma of the head and neck. Laryngoscope 1978;88:582593.

3 McNelis F, Pai V: Malignant lymphoma of the head and neck. Laryngoscope 1969;79: 1076-1087.

4 Batsakis J: Tumours of the Head and Neck. New York, Williams \& Wilkins, 1979.

$\checkmark 5$ Lang E, Walsh R, Leader M: Primary middle-ear lymphoma in a child. J Laryngol Otol 2003;117:205-207.
Solanellas J, Esteban F, Soldado L, López-Lacomba D, Hierro C, Jimenez JM: Hodgkin's disease of middle ear and mastoid. J Laryngol Otol 1996;110:869-871.

7 McCabe M, Hook CE, Burke GA: Spontaneous regression of an EBV-associated monoclonal large $B$ cell proliferation in the mastoid of a young child following surgical biopsy. Pediatr Blood Cancer 2008;51:557-559.

-8 Gapany-Gapanavicius B, Chisin R, Weshler $\mathrm{Z}$ : Primary presentation of malignant lymphoma in middle ear cleft. Ann Otol Rhinol Laryngol 1980;89:180-183.

$\checkmark 9$ Malik MK, Gupta RK, Samuel KC: Primary lymphoma of middle ear - a case report. Indian J Cancer 1976;13:188-189.
10 Danino J, Joachims HZ, Ben-Arieh Y, Hefer T, Weyl-Ben-Arush M: T cell lymphoma of the ear presenting as mastoiditis. J Laryngol Otol 1997; 111:852-854.

11 Ho TP, Carrie S, Meikle D, Wood KM: T-cell lymphoma presenting as acute mastoiditis with a facial palsy. Int J Pediatr Otorhinolaryngol 2004;68:1199-1201.

12 Laskar S, Bahl G, Muckaden M, Pai S, Nair R, Gupta S, et al: Non-Hodgkin's lymphoma of Waldeyer's ring in children: outcome using chemotherapy and involved field radiotherapy. Clin Oncol 2008;20:613-618.

13 Carbone PP, Kaplan HS, Musshoff K, Smithers DW, Tubiana M: Report of the committee on Hodgkin's disease staging classification. Cancer Res 1971;31:1860-1861. 